\title{
Survey on personality predication methods using
} AI

\author{
Jadhav Saloni, Jaras Mansi, Patil Payal, Sonawane Sunita.
}

Shatabdi Institute of Engineering \& Research, Department of Computer, Pune University, Nashik, India

\begin{abstract}
In this paper we present a deep Literature Survey on Personality. Personality is a psychological concept intended to explain the broad range of human behaviors in terms of a few, consistent and observable individual characteristics. In this regard, any technology that includes knowing, predicting and synthesizing human nature is likely to gain from technologies to Personality Computing, i.e. technologies that can deal with human character.This paper is a study of these technologies and seeks to provide not just a strong knowledge and understanding on the state-of - the art, and also a conceptual model underlying the three main issues discussed in the literature, Electronic Recognition of Character(Inference from behavioral knowledge of an individual or group true character),Automatic recognition of identities(Personality inference other people attribute to an applicant based on observable actions) and Automatic combination of identities (Artificial personality production by means of embodied agents).
\end{abstract}

Keywords-Personality prediction, AI.

\section{INTRODUCTION}

The growth of social networks is the result of social change, $t$ he sharing of information, interaction and collaboration betw een changes. On the other hand, the role of social networks in everyday life is that, and the restoration of the network of real social relationships has affected even to a large extent. At the same time, due to the easy recording, acquisition and analysis of the behavior and status of social media networks, social computing has become an important research content in the field of computer science and computers.Because the behavior and personality of people are closely linked, there is a wide intellectual value and business prospects for making personality prediction. The results show that commercial personality and commodity selection are significantly correlated.Personality data can be used extensively in personalized recommendations for ads and merchandise [1], [2].Personality is a high degree of generalization of different characteristics of mankind, even in the same situation, different people will show different characteristics that come from each person's different personality.Personality psychology is one of the branches of psychology, mainly through the outward actions of individuals to discern and analyze the relation between the intrinsic characteristics of individuals [4].Normally, psychology uses personality traits to describe the personality of individuals, describing the actions and interests of the user [5].MBTI (Myers Briggs
Type Indicator) and the big five personality model (openness, acceptability, conscientiousness, extraversion, and neuroticism) are widely used personality models. The behavior of the user's social network is closely related to his character traits. Hamburger et al. have been studying the relationship between user behavior and personality as early as 2000. Their research suggests that a correlation exists between both the personality of the user and the social services that they use. Between them, the outgoing personality is more inclined towards entertainment; the neurotic personality is less use of information services, and more popular social services [6]. [7] Used the usage habits of the keyboard and mouse to predict personality traits. Because social network user data can represent to a large extent their true personality traits, it is natural to use the social data of the user to predict personality. [9] Used the social data analysis of Facebook to assess that the extraversion and attention character had a huge positive relationship with the user's use of social network comfort. [ 10 ] By designing social networking apps on Facebook, encouraging users to participate in the big five personality test, using customer information as a data set to analyze, analysis found that the network size of the user does not reflect the social status and value of the user. To predict the relationship between user behavior and their personality traits, researchers use different research methods. Bai and others have recently released the 
state update, using a variety of machine learning algorithms such as Naive Bayesian (NB), support vector machine (SVM) and decision tree, to predict the personality of 335 users online. The results indicate that the $\mathrm{C} 4.5$ decision tree algorithm can have the greatest predictive effect and verify that the machine learning algorithm can effectively predict the personality of social media network users [11]. Verhoeven B et al. used an integrated method to determine the personality of the user to resolve the issue of incomplete training data and integrated the data of various organizational forms with the integrated method and proved the validity by experiment [12]. Through the current academic results, we may find that there is some correlation between the social media network and the personality of the user, but the use of social networks to predict the personality of the survey results of the user is relatively small. On this basis, this report examines the Sina weibo, collects the user characteristics and personality characteristics of weibo users, defines the forecasting model, analyzes and predicts the personality of the weibo user, and checks the model's feasibility.

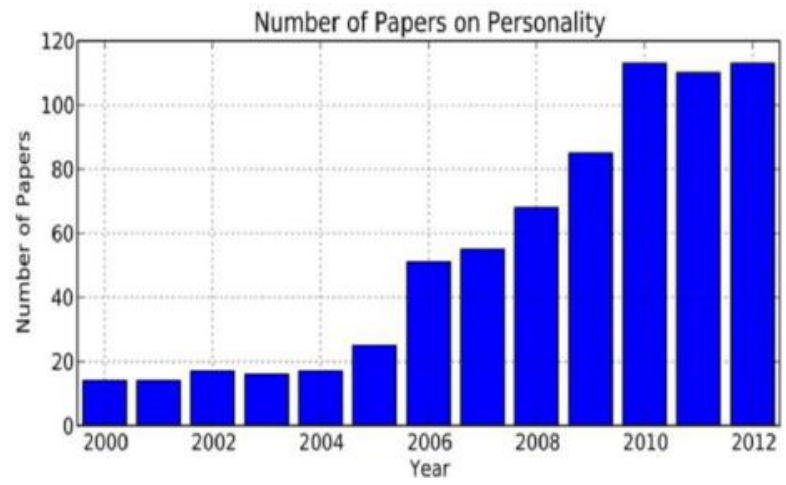

The chart reports the number of papers per year with the word "personality" in their title (sum over IEEE Explore and ACM Digital Library).

\section{LITERATURE SURVEY}

There is an increasing number of research papers in social networks linked to the actions of a consumer that has recently gained more interest in the international research community. Two key fields research the understanding of personality: computational linguistics and the evaluation of social networks. Pennebaker and King from the field of computational linguistics in 1999[14] published a pioneering work devoted to the retrieval of personality from text. In a variety of fields such as diaries, college writing tasks, and social psychology papers, they analyzed terms to analyze personality-related features with linguistic references. Their results show that pleasant people prefer to use more terms whereas introverts and low-consciousness people use more words to identify themselves. Neurotics are using more expressions of negative emotion. Argamon et al. 2005[15] identified neuroticism and extraversion using linguistic elements such as terms of action, expressions of judgment and assessment, and verbs of modality. Their findings showed that neuroticism is related to the use of functional lexical features, such as lexical taxonomy evaluation, whereas the results for extraversion were less evident. Many studies linked neuroticism to irrational beliefs or weak coping efforts on personality well-being [16] and Nowson 2006 categorized bloggers' extraversion, resilience, reliability, and conscientiousness using the Naive Bayes prediction model as a learning algorithm using various sets of n-grams as features. Mairesse et al. 2007[17] investigated correlations between the characteristics of the Big 5, using LIWC and RMC as feature sets. While LIWC features included word identification such as positive feelings or frustration, RMC features included word age results or word imageability results. Mairesse et al. developed a controlled program of personality identification using the corpus of essays written by Pennebaker and King in 1999. Of Social Network Research, there is an even short history of personality identification from network setup and other extralinguistic signals. Gosling et al. studied the effect of the social interaction behavior of a user on personality [21]. They studied personality traits from self-reported use of Facebook and observable information on the profile. Rather than just emotional properties, all interface interfaces are based on statistical characteristics. Bacharach et al. [22] found that from their Facebook profiles, people can judge the personalities of others. Golbeck and so on. [23] Forecast the personality of 279 Facebook users using language features such as word count and social media site features such as counting friends. Orr and others, 2009 [24] showed a positive correlation between shyness and time spent online and a negative correlation with the number of friends. Sumner et al.[25] established the connection between the personalities of users and their use of Facebook, sharing material and emotion. Their result showed that transparency is positively associated with words that express negative feelings, rage, tabo subjects, income, religion, or death. Kalish and Robins [26] experimentally investigated the impact of behavior differences between individuals on their immediate network environment, focusing on ego networks consisting of a focal node or ego and the nodes to which the ego is directly connected (the so-called alters) and, if any, the connections between the alters. Their results indicated that the variability 
portions of egocentric network features can be clarified by psychological predispositions. Personality prediction based on vocabulary characteristics has been highly interested in prediction research [27]-[29], but from a network perspective, the role of links in supporting personality relationships is not yet well recognized.

Our research aims to investigate the existence of a social media structure and linguisticcharacteristics linked to personality experiences using the myPersonality project dataset [30].

\section{DATASETS}

We used the myPersonality dataset as a case study to analyze the personality traits of social media. We built the analysis fr om the myPersonality survey with 250 users and 9917 status updates.Facebook users ' dataset was named in keeping with $t$ he Big 5 template. According to the personality styles distrib ution in Table 3, multiple posts are collected in one folder by each client in the dataset [30].

\section{* TABLE:}

Distribution of Personality Traits

\begin{tabular}{|l|l|l|l|l|l|}
\hline value & o & c & e & a & n \\
\hline Yes & 176 & 130 & 96 & 134 & 99 \\
\hline No & 74 & 120 & 154 & 116 & 151 \\
\hline
\end{tabular}

* Note:-

$\mathrm{O}, \mathrm{C}, \mathrm{E}, \mathrm{A}, \mathrm{N}$ applies to Facebook's distribution of perso nality ratings on the Big Five traits: openness, intelligenc e, extraversion, congeniality, and neuroticism.

We picked user information such as user social network layout, user profile, and text messages with some of the dataset. The final dataset contains the raw text Facebook status, author information, personality labels (scores and classes) and five user social media network metrics inferred by personality characteristics, i.e. network size, betweeness, distance, brokerage, and transitivity.

\section{DISCUSSION}

Through the current academic findings, we can find that there is some connection between the social network and the personality of the user, but the use of social media sites tothe personality of the survey results of the user is relatively small. This paper examines the Sinaweibo on the basis of these, extracts the user's characteristics and personality characteristics of weibousers, develops the predictive model, examines and predicts the personality of the weibo user and verifies the feasibility of the model.

\section{CONCLUSION}

Based on the existing research, we focus on the characteristics of the social network and user behavior and set up three models for comparative analysis. We found that the behavioral characteristics of the user and their personality are related by studying social network data can effectively predict the personality characteristics of the user, which we can concentrate on the characteristics of social network and user activity on the basis of existing research, and set up three predictive models for comparative analysis. We found that, through the analysis of social network data, the behavioral characteristics of the user and their personality are related can be largely predictive of the personality characteristics of the user, which can urtherly facilitate the creation of customized applications. Use AI, more accurate framework for personality prediction can be built in the future.

\section{REFERENCES}

[1] G. Odekerken-Schrder, K. D. Wulf, and P. Schumacher, "Strengthening outcomes of retailer consumer relationships: The dual impact of relationship marketing tactics and consumer personality," Journal of BusinessResearch, vol. 56, no. 3, pp. 177-190, 2003.

[2] S. Whelan and G. Davies, "Profiling consumers of own brands andnational brands using human personality," Journal of Retailing andConsumer Services, vol. 13, no. 6, pp. 393402, 2006.

[3] P. J. Rentfrow and S. D. Gosling, "The do re mi's of everyday life: Thestructure and personality correlates of music preferences." Journal ofPersonality and Social Psychology, vol. 84, no. 6 , p. 1236, 2003.

[4] G. Matthews, I. J. Deary, and M. C. Whiteman, "Personality traits (2nd.ed.)." 2003.

[5] G. W. Allport, "The general and the unique in psychological science."Journal of Personality, vol. 30, no. 3, p. 405422, 1962.

[6] Y. A. Hamburger and E. Ben-Artzi, "The relationship between extraversionand neuroticism and the different uses of the internet," Computersin Human Behavior, vol. 16, no. 4, pp. 441-449, 2000.

[7] Khan, W.-P. Brinkman, N. Fine, and R. M. Hierons, "Measuringpersonality from keyboard and mouse use." ACM, 2008, p. 38. 
[8] Marcus, F. Machilek, and A. Schtz, "Personality in cyberspace: Personalweb sites as media for personality expressions and impressions. "Journal of Personality and Social Psychology, vol. 90, no. 6, pp. 1014-31, 2006”.

[9] Ross, E. S. Orr, M. Sisic, J. M. Arseneault, M. G. Simmering, andR. R. Orr, "Personality and motivations associated with Facebookuse, "Computers in Human Behavior, vol. 25, no. 2, pp. 578-586, 2009.

[10] J. Golbeck, C. Robles, and K. Turner, "Predicting personality with socialmedia," 2011, pp. 253-262.

[11] C. L. Bai S, Zhu T, "Big-five personality prediction based on user behaviors at social network sites," Plos Neglected Tropical Diseases,vol. 8, no. 2, p. 2682, 2012.

[12] B. Verhoeven, W. Daelemans, and T. D. Smedt, "Ensemble methods forpersonality recognition," 2013, pp. 35-38.

[13] J. Staiano, B. Lepri, N. Aharony, F. Pianesi, N. Sebe, and A. Pentland,"Friends don't lie: inferring personality traits from social networkstructure," in Proceedings of the 2012 ACM conference on ubiquitouscomputing. ACM, 2012, pp. 321330.

[14] J. W. Pennebaker, R. L. Boyd, K. Jordan, and K. Blackburn, "The developmentand psychometric properties of liwc2015," Tech. Rep., 2015.

[15] S. Argamon, S. Dhawle, M. Koppel, and J. Pennebaker, "Lexical predictorsof personality type," 2005.

[16] R. R. McCrae and P. T. Costa, "Personality, coping, and coping effectivenessin an adult sample," Journal of personality, vol. 54, no. 2, pp. 385-404,1986.

[17] B. R. Karney and T. N. Bradbury, "The longitudinal course of marital qualityand stability: A review of theory, methods, and research." Psychologicalbulletin, vol. 118, no. 1, p. 3, 1995.

[18] P. T. Costa and R. R. McCrae, "Normal personality assessment in clinicalpractice: The neo personality inventory." Psychological assessment, vol. 4,no. 1, p. 5, 1992.

[19] S. Adali and J. Golbeck, "Predicting personality with social behavior,"in Advances in Social Networks Analysis and Mining (ASONAM), 2012IEEE/ACM International Conference on. IEEE, 2012, pp. 302-309.

[20] O. P. John and S. Srivastava, "The big five trait taxonomy: History, measurement,and theoretical perspectives," Handbook of personality: Theoryand research, vol. 2, no. 1999, pp. 102-138, 1999.

[21] D. Gosling, A. A. Augustine, S. Vazire, N. Holtzman, and S. Gaddis,"Manifestations of personality in online social networks: Self-reportedFacebook-related behaviors and observable profile information, "cyber psychology, Behavior, and Social Networking, vol. 14, no. 9, pp.483-488, 2011.

[22] H. Davis, H. Halpin, A. Pentland, M. Bernstein, L. Adamic, H. Alani,A. Monnin, and R. Rogers, Proceedings of the 3rd ACM Web ScienceConference, 2013 Paris, France, WebSci'13. ACM, 2013.
[23] J. Golbeck, C. Robles, and K. Turner, "Predicting personality with socialmedia," in CHI'11 extended abstracts on human factors in computingsystems. ACM, 2011, pp. 253-262.

[24] C. Ross, E. S. Orr, M. Sisic, J. M. Arseneault, M. G. Simmering, andR. R. Orr, "Personality and motivations associated with facebookuse,"Computers in human behavior, vol. 25, no. 2, pp. 578-586, 2009.

[25] C. Sumner, A. Byers, and M. Shearing, "Determining personality traits \&privacy concerns from Facebookzz activity,” Black Hat Briefings, vol. 11, pp.197-221, 2011.

[26] Y. Kalish and G. Robins, "Psychological predispositions and networkstructure: The relationship between individual predispositions, structuralholes and network closure," Social networks, vol. 28, no. 1, pp. 56-84, 2006.

[27] F. Iatan, "Predicting human personality from social media using a fuzzyneural network," in Issues in the Use of Neural Networks in InformationRetrieval. Springer, 2017, pp. 81105.

[28] H. A. Schwartz, J. C. Eichstaedt, M. L. Kern, L. Dziurzynski, S. M.Ramones, M. Agrawal, A. Shah, M. Kosinski, D. Stillwell, M. E. Seligmanet al., "Personality, gender, and age in the language of social media: Theopen-vocabulary approach," PloS one, vol. 8, no. 9, p. e73791, 2013.

[29] T. Yarkoni, "Personality in 100,000 words: A large-scale analysis of personalityand word use among bloggers," Journal of research in personality,vol. 44, no. 3, pp. 363-373, 2010.

[30] M. Kosinski, S. C. Matz, S. D. Gosling, V. Popov, and D. Stillwell, "Facebookas a research tool for the social sciences: Opportunities, challenges, ethical considerations, and practical guidelines." American Psychologist,vol. 70, no. 6, p. 543, 2015. 